\title{
Foco de leishmaniasis en El Hobo, municipio de El Carmen de Bolívar, Bolívar, Colombia
}

\author{
Luis Alberto Cortés
}

Unidad de Entomología, Laboratorio de Salud Pública Departamental, Secretaría de Salud de Bolívar, Cartagena, Colombia.

Introducción. Se describen las características epidemiológica e importancia de la especies de Lutzomyia presentes en un foco de leishmaniasis en la vereda El Hobo Carmen de Bolívar, departamento de Bolívar.

Objetivos. Obtener un conocimiento preliminar de la transmisión de leishmaniasis en la vereda El Hobo Carmen de Bolívar.

Materiales y métodos. Se analizaron datos epidemiológicos y se realizaron capturas de flebótomos con trampas CDC y cebo humano en la vereda El Hobo. Para establecer la sero prevalencia de leishmaniasis visceral canina se hizo un estudio en perros mediante la técnica de inmunofluorescencia indirecta.

Resultados. Se capturaron nueve especies de Lutzomyia: L. trinidadensis, L. evansi, L .cayennensis, L. venezuelensis, L. gomezi, L. dubitans, L. ylephiletor, L. yuilli, y L. walkeri. Las especie de mayor importancia por sus implicaciones en la transmisión de leishmaniasis cutánea y visceral fueron L. gomezi, y L. evansi respectivamente. Se reporta por primera vez para Bolívar especimenes de L. venezuelensis, L. dubitans, L. ylephiletor, L. yuilli, y L. walkeri. Se determinó una prevalencia de leishmaniasis visceral del $36 \%$ en los caninos estudiados. Según los reportes epidemiológicos, en el 2002 en el municipio de Carmen de Bolívar la leishmaniasis cutánea mostró un aumento del $40 \%$ y la leishmaniasis visceral canina del $80 \%$ de los casos con respecto al 2001, debido al brote presentado en la vereda El Hobo

Conclusiones. Los resultados determinan a la vereda El Hobo como una zona de riesgo potencial de transmisión de leishmaniasis cutánea y visceral.

Palabras clave: Leishmania, Lutzomyia, leishmaniasis visceral, leishmaniasis cutánea, reservorios, epidemiología.

Leishmaniasis transmission focus in El Hobo, Carmen de Bolívar, Bolívar, Colombia)

Introduction. The epidemiological characteristics and the importance of the Lutzomyia species present in a leishmaniasis focus in the village El Hobo, Carmen de Bolívar, Department of Bolívar, Colombia, are described..

Objective. To obtain a preliminary assessment of leishmaniasis transmission in the village of El Hobo, Carmen de Bolívar.

Materials and methods. Epidemiological data were analyzed and sandflies were collected with CDC traps and on protected human volunteers in the village of El Hobo. Sero prevalence of canine visceral leishmaniasis in dogs was evaluated using indirect inmunofluorescence (IFAT).

Results. Nine Lutzomyia species were collected: L. trinidadensis, L. evansi, L. cayennensis, $L$. venezuelensis, L. gomezi, L. dubitans, L. ylephiletor, L. yuilli, and L. walkeri. The species of greater importance based on their implication in the transmission of cutaneous and visceral leishmaniasis were $L$. gomezi, and $L$. evansi respectively. Specimens of $L$. venezuelensis, $L$. dubitans, L. ylephiletor, L. yuilli, and L. walkeri are reported for the first time for the Department of Bolivar. The sero prevalence of visceral leishmaniasis in dogs was $36 \%$. According to the epidemiological records, in 2002 the municipality of Carmen de Bolivar presented a $40 \%$ increase in cases of cutaneous leishmaniasis and an $80 \%$ increase in canine visceral leishmaniasis as compared to 2001 , due to the outbreak in the village of El Hobo. 
Conclusions. The results indicate that the village of El Hobo is an area of potential risk for transmission of both cutaneous and visceral leishmaniasis.

Key words: Leishmania, Lutzomyia, visceral leishmaniasis, cutaneous leishmaniasis, reservoirs, epidemiology.

Todas las leishmaniasis son trasmitidas al hombre únicamente por la picadura de especies de flebótomos que, en el Nuevo Mundo pertenecen al genero Lutzomyia y en el Viejo Mundo al género Phlebotomus (1).Cada especie de Leishmania tiene un único perfil epidemiológico con diferentes vectores, huéspedes, reservorios y distribución geográfica. La mayoría de casos humanos ocurre en personas que residen o entran a áreas rurales o selváticas ya que la enfermedad es básicamente una zoonosis (2). La epidemiología de estas enfermedades es extremadamente compleja y puede ser alterada por cambios en algún punto del triángulo epidemiológico que está formado por humanos, reservorios y flebótomos transmisores. La mayoría de los factores ambientales que afectan la epidemiología de varias leishmaniasis son aún pobremente entendidos o no se conocen muy bien (3). En el 2002, Carmen de Bolívar aportó 187 casos de leishmaniasis cutánea y 19 casos de leishmaniasis visceral, el $63 \%$ y el $99 \%$ de los casos en el departamento de Bolívar, respectivamente. La vereda El Hobo en el 2002 presentó 76 casos de leishmaniasis cutánea y 17 de los 19 casos reportados de leishmaniasis visceral en el municipio Carmen de Bolívar (datos sin publicar, Secretaría de Salud de Bolívar. Informes de entomología, ETV y epidemiología, 2003). De acuerdo con estos antecedentes epidemiológicos, la Unidad de Entomología de la Secretaría de Salud Departamental decidió realizar un estudio que tenía como objetivo determinar las características ecoepidemiológicas del foco y las especies de Lutzomyia presentes que permitieran dilucidar el ciclo de transmisión de la enfermedad y poder

\footnotetext{
Correspondencia:

Luis Alberto Cortés, coordinador, Unidad de Entomología, Laboratorio de Salud Pública Departamental, Secretaría de Salud de Bolívar, Centro calle 36 №7-55, Casa de la Moneda, Cartagena, Colombia.

Teléfono fax (095) 6643600

luisgonatodes@yahoo.com. luisgonatodes@hotmial.com.

Recibido: 30/06/05; aceptado: 02/02/06
}

elaborar métodos de control y prevención eficaces en la vereda El Hobo.

\section{Materiales y métodos}

Área de estudio y población. El estudio se llevó a cabo en la vereda El Hobo, municipio de Carmen de Bolívar, departamento de Bolívar.

Investigación epidemiológica. Se analizaron los datos de las fichas epidemiológicas de leishmaniasis de los pacientes procedentes de la vereda El Hobo del 2002 y se compararon con los del 2001. Estas fichas se hallaban en la Secretaría de Salud Municipal de Carmen de Bolívar y la Secretaría de Salud Departamental. Se tomaron como variables relevantes la edad y el sexo del paciente.

Investigación entomológica. El muestreo de flebótomos se realizó del 15 al 18 de enero del 2003. Se seleccionó una casa en la vereda El Hobo en donde se habían presentado dos casos de leishmaniasis visceral y uno de cutánea. Se muestreó con trampa de luz CDC por dos noches en el intradomicilio, y una noche en el peridomicilio y extradomicilio por espacio de 12 horas de las 17:00 a las 05:00 horas y se muestreó en cebo humano protegido en el intradomicilio durante tres noches de las 17:00 a las 24:00 horas. Para tal efecto, dos personas con protección adecuada, camisa de manga larga y pantalón largo, y con repelente en las partes expuestas, se sentaron a esperar la llegada de los insectos.

Se hizo búsqueda activa en el intradomicilio, en las paredes de la vivienda de las 17:00 a las 20:00 horas. Las capturas las hicieron cuatro recolectores, con la ayuda de una linterna y un aspirador bucal. Se registró el número de individuos por hora; los insectos capturados se asfixiaron con humo y se preservaron en alcohol al $70 \%$, debidamente rotulados para ser enviados al Laboratorio de Entomología del Instituto Nacional de Salud y a la Unidad de Entomología de Bolívar para su determinación taxonómica 
según las claves taxonómicas de Young y Duncan (4).

Encuesta serológica. Para determinar la prevalencia de leishmaniasis canina mediante la técnica inmunofluorescencia indirecta (IFI) se capturaron perros en la vereda El Hobo, a los cuales se les extrajeron $5 \mathrm{ml}$ de sangre en tubos (vacutainer) por venopunción. Luego, se separó el suero por centrifugación a 5000 rpm durante 5 minutos y se conservó en frío hasta su procesamiento.

La captura de los caninos y la recolección de las muestras, al igual que las capturas en cebo humano, fueron aprobadas por el Comité de Zoonosis conformado por el ICA y la Secretaría de Salud de Bolívar.

En la vereda El Hobo se estimaba un total de 48 perros, pero sólo se les pudo tomar muestra de sangre a 36 , encontrados en las casas visitadas en la vereda. Los exámenes realizados a las personas y a los caninos mediante la técnica IFI para el diagnóstico de leishmaniasis visceral fueron bajo los parámetros del Laboratorio de Parasitología del Instituto Nacional de Salud. Se utilizó el antígeno cepa Leishmania chagasi, suministrado por el Instituto Nacional de Salud, y se consideraron positivos los resultados mayores o iguales a 1/32 (comunicación personal con colaboradores del INS).

Análisis estadístico. Se realizó un análisis descriptivo de la población con las variables edad y sexo; se utilizó una prueba no paramétrica $G$, para determinar diferencias significativas entre la presencia de leishmaniasis por sexo y edades.

Se determinó la prevalencia de leishmaniasis visceral canina en perros de la vereda El Hobo. Los datos se analizaron con el programa SPSS para Windows.

\section{Resultados}

Según los reportes epidemiológicos, en el 2002 en el municipio de Carmen de Bolívar la leishmaniasis cutánea mostró un aumento del $40 \%$ y la leishmaniasis visceral canina del $80 \%$ de los casos con respecto al 2001, debido al brote presentado en la vereda El Hobo. Mediante la prueba no paramétrica $G$ no se encontraron diferencias significativas entre hombres y mujeres que presentaron leishmaniasis cutánea $\left(G_{0,05,1=4.9,}\right.$, $0>0,95)$ como tampoco en el rango de edades tomado $(0-10,11-20,21-35,<36),\left(G_{0,05,1=1,3, p>0,08}\right)$. Para leishmaniasis visceral se encontró el mayor número de casos entre cero y dos años de edad. A través del 2002 los casos de leishmaniasis cutánea y visceral se presentaron en todos los meses del año, con un leve aumento en los meses de noviembre y diciembre.

En cuanto al muestreo de Lutzomyia, se capturaron 190 individuos de Lutzomyia, 73 por trampas CDC, 107 en cebo humano y 10 en búsqueda activa. Del total de ejemplares capturados, 68\%(130) eran hembras y $30,52 \%$ (60) machos; de éstos, 55 fueron capturados por trampas CDC, 2 por búsqueda activa y 3 en cebo humano. Del total de hembras, 94 fueron por cebo humano, 28 en trampa CDC y 8 por búsqueda activa.

Según los sitios -intradomicilio, peridomicilio y extradomicilio- se observó que la mayor captura ocurrió en el extradomicilio con una densidad de 39 Lutzomyia por trampa por 1 noche, seguida por el intradomicilio con una densidad de 9 Lutzomyia por trampa por noche y el peridomicilio que tuvo una densidad muy baja, 1 Lutzomyia por trampa por noche. La tasa de picadura en el intradomicilio mostró actividad desde las 17:00 a las 21:00 horas, con un pico máximo entre las 21:00 y las 22:00 horas con una densidad de 6 Lutzomyia/hora/hombre (figura 1).

Las especies de Lutzomyia capturadas fueron: $L$. trinidadensis, L. evansi, L. cayennensis, $L$. venezuelensis, L. gomezi, L. dubitans, $L$. ylephiletor, L. yuilli y L. walkeri.

De los 36 perros muestreados, 13 resultaron positivos para leishmaniasis visceral canina con una prevalencía del $36 \%$.

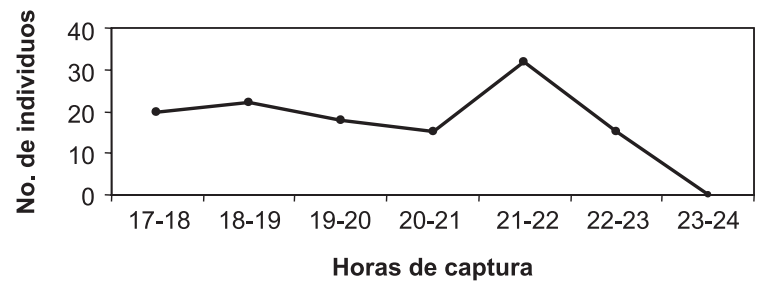

Figura 1. Horas de actividad de Lutzomyia spp. en el intradomicilio, vereda El Hobo. 


\section{Discusión}

La vereda El Hobo queda en la zona rural del municipio El Carmen de Bolívar; se ubica entre un bosque secundario con algunos parches de bosques secos tropicales, comparte fronteras con veredas del departamento de Sucre en donde existe un foco histórico de leishmaniasis visceral (5). Para el caso de las leishmaniasis, el desarrollo urbano en áreas endémicas crea condiciones de traslape en donde la vivienda humana se aproxima a los focos naturales con la consecuente aparición de individuos infectados (6). La presencia de individuos de diferentes edades entre un mes y 78 años al igual que de ambos sexos, sugiere una transmisión intradomicilio y peridomicilio de leishmaniasis cutánea en la vereda El Hobo. Los resultados de prevalencia de caninos positivos por IFI comparados con otros reportes realizados en la región, mostraron una seroprevalencia conforme a lo registrado en San Andrés de Sotavento, Córdoba, 16\% por IFI (7) y más altos que los reportados por Ovejas, Sucre, y El Carmen de Bolívar, 9,6\% (5), La seroprevalencia encontrada es alta comparada con recientes estudios realizados en el mundo: en España, 4,5\% (8); Brasil, 6,2\% (9), e Irán, 18\% (10).

Los estudios de la dinámica de la población de especies de Lutzomyia involucradas en la transmisión de la leishmaniasis en áreas rurales y suburbanas, son importantes para el conocimiento de los factores bióticos y abióticos que mantienen estas parasitosis como una enfermedad endémica en zonas rurales y se constituyen en estudios de gran valor para la planificación de las estrategias de control vectorial (11).

A pesar del corto tiempo del estudio, el presente trabajo permite tener una buena aproximación a la composición de especies de Lutzomyia implicadas en un foco de leishmaniasis en la vereda El Hobo. Se observaron nueve especies del género Lutzomyia, lo cual aumenta de 13 a 18 el número de especies reportadas para Bolívar según Ferro y Morales en 1998 (12).

Se han reportado para Colombia 129 especies de Lutzomyia (13), sólo siete de ellas se han incriminado como vectores: $L$. longipalpis, $L$. evansi, L. spinicrassa, L. hartmanni, L. trapidoi, L. flaviscutellata y L. gomezi (14). De las especies encontradas en este estudio las de mayor importancia por sus antecedentes como vectores eran L. gomezi, vector reconocido de leishmaniasis cutánea $(12,13,15)$ y $L$. evansi, vector principal de la leishmaniasis visceral en la costa del Caribe colombiano (4,16-18).

Epidemiológicamente es importante reconocer el comportamiento y los hábitos de picadura de las Lutzomyia, para establecer las horas y los sitios donde se encuentran expuestos los habitantes de una región determinada $(6,15)$. De acuerdo con las capturas hechas en cebo humano en el intradomicilio, las especies de Lutzomyia muestran una actividad entre las cinco de la tarde y las once de la noche (figura 1), sobreponiéndose con las actividades humanas como descansar, ver televisión, oir radio, o dormir; esto puede explicar, hasta cierto punto, la gran incidencia de la enfermedad, además del mal manejo del medio y la poca protección contra los vectores, motivo del desconocimiento de las comunidades afectadas acerca de la enfermedad y el vector (datos epidemiológicos sin publicar, Unidad de Entomología de Bolívar).

Después de analizar los datos de este estudio la Secretaría de Salud decidió tomar como medidas de control y prevención la utilización de toldillos impregnados en las viviendas dispersas, fumigación de acción residual en las viviendas conglomeradas y educación a la comunidad sobre los métodos de prevención y control de la enfermedad. Aunque es muy difícil el seguimiento de estas actividades por el delicado acceso a la vereda, se puede decir que los métodos utilizados fueron eficaces, dado que la vereda El Hobo no ha reportado casos de leishmaniasis cutánea ni visceral desde el segundo semestre del 2003 (datos sin publicar, Secretaría de Salud de Bolívar, datos de entomología, ETV y Epidemiología, 2004).

En conclusión, en el presente trabajo se reportan cinco nuevos registros de Lutzomyia para el departamento de Bolívar: $L$. venezuelensis, $L$. dubitans, L. ylephiletor, L. yuilli, y L. walkeri.

Teniendo en cuenta la presencia de $L$. evansi, y L. gomezi, vectores principales de leishmaniasis 
cutánea y visceral en la costa Caribe colombiana, la vereda El Hobo constituye una zona de riesgo potencial de transmisión de leishmaniasis cutánea y visceral.

Si se tiene en cuenta que el reservorio de la leishmaniasis visceral es el perro, y que se da un intercambio importante de diferentes tipos, como transporte de mascotas por las personas desplazadas por la violencia, visita de familiares $y$ actividades de comercio entre las veredas de los departamentos de Sucre y Bolívar en donde los focos de leishmaniasis visceral están activos, es muy difícil controlar la endemia si no se pone todo el empeño de las autoridades municipales y departamentales de salud.

De acuerdo con los datos arrojados por este estudio preliminar se hacen necesarios estudios individuales de los diferentes focos presentes en la región, así como un estudio más profundo y detallado de las especies $L$. gomezi y $L$. evansi para poder determinar efectivamente las medidas control y prevención de la leishmaniasis visceral y cutánea de acuerdo con las características epidemiológicas y ecológicas de cada vereda en el municipio de El Carmen de Bolívar.

\section{Agradecimientos}

A Margarita Martínez y a Martha Hernández por su apoyo a la logística del estudio. A Alcira Castro por los datos epidemiológicos, a Yonia Cabarcas por su apoyo en la toma de muestras. A Ramiro Pereira y Guillermo Laguna por su invaluable apoyo en campo. A Adán Márquez y a todos los técnicos del municipio de El Carmen de Bolívar que participaron en la realización de este proyecto. A Víctor Olano, Cristina Ferro y Erika Santamaría por su colaboración en la determinación taxonómica de los individuos recolectados en el Laboratorio de Entomología del Instituto Nacional de Salud. A Martha Ayala por su apoyo y la realización de los IFI en el Laboratorio de Parasitología del Instituto Nacional de Salud.

\section{Conflicto de intereses}

Se manifiesta que los resultados obtenidos en esta investigación no están relacionados con ningún tipo de intereses.

\section{Financiación}

Este estudio pertenece a los Programas de Prevención y Control de ETV de la Secretaría de Salud de Bolívar, y fue financiado por la Gobernación de Bolívar, Secretaría de Salud.

\section{Referencias}

1. Desjeux P. Human leismaniases: epidemiology and public health aspects. Word Health Stat Q 1992;45:26775.

2. Grimaldi G Jr, Tesh RB. Leishmaniasis of the New World: current concepts and implications for future research. Clin Microbiol Rev 1993;6:230-50.

3. Sandoval CM, Angulo VM, Gutiérrez R. Muñoz G, Ferro C. Especies de Lutzomyia (Diptera: Psychodidae) posibles vectores de leishmaniasis en la ciudad de Bucaramanga, Santander, Colombia. Biomédica 1998;18:161-8.

4. Young DG, Duncan MA. Guide to the identification and geographic distribution of Lutzomyia sandflies in Mexico, the West Indies, Central and South America (Diptera Psychodidae). Mem Am Entomol Inst 1994;54:1-881.

5. Fernández J, Charry TA, Bello F, Escovar J, Lozano C, Ayala M et al. Prevalencia de leishmaniosis visceral canina en municipios de Huila, Colombia. Rev Salud Pública 2002;4:278-85.

6. Scorza JV, Macias P, Rojas J. Encuestas epidemiológicas sobre leishmaniasis cutánea urbana en la ciudad de Trujillo, Venezuela. Bol Dir Mal San Abm 1985;2:389-404.

7. Vélez ID, Travi BL, Gallego J, Palma GL, Agudelo SP, Montoya J. Evaluación ecoepidemiológica de la leishmaniosis visceral en la comunidad indígena Zenu de San Andrés de Sotavento, Córdoba: primer paso para su control. Revista Colombiana de Entomología 1995;21:111-2.

8. Sanchis MC, Martín J, Amate P, Acedo C, Miras N, Mostpha L et al. Estudio epidemiológico de la leishmaniosis en Almería, España. Pharmaceutica 1997;38:53-61.

9. Silva ES, Gantijo CM, Santos SG, Amorin VD, Lemos FL, Primes C et al. Visceral leishmaniasis in Riberao das Neves, municipality of Metropolitan Region of Belo Horizonte MG Brasil. Mem Inst Oswaldo Cruz 1998;93(Suppl.11):138-9.

10. Sharifi I, Deneshvar H. The prevalence of visceral Leishmaniasis in suspected canine reservoirs in southeastern Iran. Am J Trop Med Hyg 1996;21:3-4.

11. Perruolo G. Aspectos ecológicos de Lutzomyia spp. (Diptera: Psychodidea) en un foco endémico de leishmaniasis cutánea en el estado Táchira, Venezuela. Bol Malariol Sal Amb 2004; XLIV:35-44. 
12. Ferro C, Morales A. Flebótomos de Colombia. Estudios realizados por el Laboratorio de Entomología. INS, 19651997. En: Toro G, Hernández CA, Raad J, editores. Instituto Nacional de Salud 1917-1997. Una historia un compromiso. Santa Fe de Bogotá: Instituto Nacional de Salud; 1998.

13. Montoya J, Ferro C. Flebótomos (Diptera:Psychodidae) de Colombia. En: Amat G, Andrade MG, Fernández F, editores. Insectos de Colombia. Volumen II. Colección Jorge Álvarez Lleras No. 13. Academia Colombiana de Ciencias Exactas, Físicas y Naturales. Santa Fe de Bogota: Centro Editorial Javeriano; 1999. p.211-45.

14. Wolf M, Sierra D, Murcia LM, Vélez ID. Phlebotominae fauna (Diptera: Psychodidae) in the Department of Amazonas, Colombia. Neotrop Entomol 2003;32:523-6.
15. Zeledón R, Murillo J, Gutiérrez H. Flebótomos antropofílicos y leishmaniasis cutánea en Costa Rica. Bol Of Sanit Panam 1985;99:163-72.

16. Gallego JL, Vélez ID. Presencia en Isla Fuerte, Bolívar, de Lutzomyia evansi, vector de leishmaniasis visceral. latreia 1994;7:33-5.

17. Travi BL, Montoya J, Gallego J, Jaramillo C, Llano R, Vélez ID. Bionomics of Lutzomyia evansi (Diptera: Psychodidae), vector of visceral leishmaniasis in northern Colombia. J Med Entomol 1996;33:278-85.

18. Adier GH, Becerra MT, Travi BL. Feeding success of Lutzomyia evansi (Diptera: Psychodidae) experimentally exposed to small mammal hosts in an endemic focus of Lehismania chagasi in northern Colombia. Biomédica 2003;23:396-400. 